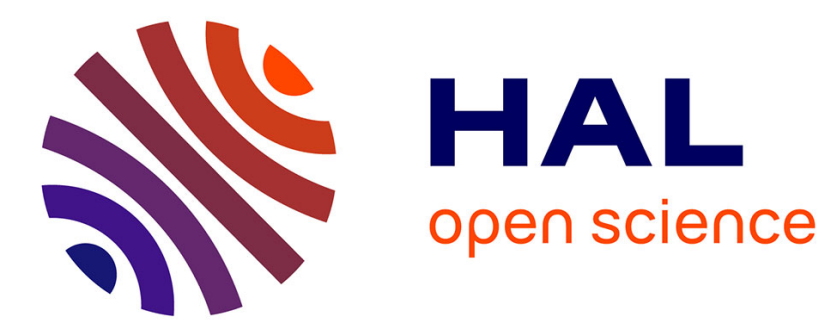

\title{
Comment nourrissait-on les oiseaux dans la rotonde de la volière de Varron (Rust. 3, 5, 4)?
}

Ginette Vagenheim

\section{To cite this version:}

Ginette Vagenheim. Comment nourrissait-on les oiseaux dans la rotonde de la volière de Varron (Rust. 3, 5, 4) ?. Rivista di Filologia e d'Istruzione Classica, 2017, 147 (2), pp.394-399. hal-01827010

\section{HAL Id: hal-01827010 https://hal.science/hal-01827010}

Submitted on 9 Aug 2018

HAL is a multi-disciplinary open access archive for the deposit and dissemination of scientific research documents, whether they are published or not. The documents may come from teaching and research institutions in France or abroad, or from public or private research centers.
L'archive ouverte pluridisciplinaire HAL, est destinée au dépôt et à la diffusion de documents scientifiques de niveau recherche, publiés ou non, émanant des établissements d'enseignement et de recherche français ou étrangers, des laboratoires publics ou privés. 


\title{
COMMENT NOURRISSAIT-ON LES OISEAUX DANS LA ROTONDE DE LA VOLIÈRE DE VARRON (RUST. 3, 5, 4)?
}

\begin{abstract}
This article proposes a new interpretation of part of Varro's description of his aviary, i.e. rust. 3, 5, 14-15. More precisely, it considers the method of feeding the birds in the cage in the tholus, which is different from the method of feeding them in the cages in the porticoes (rust. $3,5,11$ ). In the cages in the porticoes, the birds are fed per retem, while in the cage in the tholus they receive their food sub retem. The reason for this difference is to be found in the materials chosen for building the cages and the reasons for these choices.
\end{abstract}

Keywords: Roman villa, Varro' res rusticae book III, Varro's birdhouse, Casinum, Roman architecture, luxus.

Malgré les nombreuses tentatives de représenter la volière que Varron fit construire à Casinum pour son plaisir (animi causa, 3, 5, 5) et qu'il décrit au livre III des res rusticae ${ }^{1}$, il reste encore certains détails techniques, dans la construction de l'édifice, qui ont totalement échappé à l'attention des critiques; il s'agit notamment du système d'alimentation en eau et en nourriture dans les divers habitats des volatiles. Dans un article précédent, j'ai proposé une nouvelle interprétation du système d'alimentation en eau destiné aux oiseaux enfermés dans les deux cages en forme de portiques: l'examen des premières éditions imprimées des Res rusticae (1472-1595) m'a permis de rétablir la division correcte du texte et de montrer ainsi que la description de la petite rigole (rivolo tenui) ne s'arrête pas au livre 3, 5, 11, comme l'indiquent toutes les éditions modernes ${ }^{2}$, mais se

${ }^{1}$ On citera, entre autres, Van Buren - Kennedy 1919, 59-66; Des Anges - Seure 1932 et Fuchs 1962, 96-105.

2 Les éditions modernes principales se fondent sur l'editio minor de Keil 1902; elle est reprise par Traglia 1979, par Hoope - Ash 1934 et dans l'édition de Guiraud 1997. Pour la bibliographie très vaste relative à la volière de Varron, voir Sauron 1994; Dunbabin 1996, 66-80; Green 1997, 
poursuit jusqu'au $\S 12$; on comprend ainsi que la petite rigole, probablement alimentée par l'eau du fleuve tout proche, courait tout le long du stylobate intérieur de la colonnade (secundum stylobatis interiorem partem), dans chaque portique (dextra et sinistra), sur toute la longueur de l'aire rectangulaire (ad summam aream quadratam). Dans la présente étude, je m'intéresserai au mode de distribution de la nourriture dans la cage de la rotonde.

$\mathrm{Au}$ moment d'évoquer la cage située entre les deux colonnades de la rotonde, Varron suit rigoureusement le même ordre que la description des cages des portiques; il en décrit d'abord l'architecture $(3,5,13)$, puis le genre des oiseaux qui y sont enfermés et enfin leur mode d'alimentation en eau et en pâture $(3,5,14)$; ainsi, dans la rotonde, l'eau arrive par un petit canal (canaliculum, 3, 5, 14) dont on ignore s'il était lui aussi directement relié au fleuve, comme la petite rigole des portiques ou si son alimentation exigeait une intervention extérieure; c'est ce que semble sous-entendre le verbe ministratur, utilisé au passif indéfini «quibus aqua ministratur per canaliculum»; en effet, c'est exactement la même forme qui est utilisée pour désigner que quelqu'un se charge de nourrir les oiseaux dans les cages des portiques, en leur passant la pâture à travers les mailles du filet de chanvre $(3,5,11$ : quibus cibus ministratur per retem)

Dans la rotonde, en revanche, la nourriture n'était pas donnée aux oiseaux à travers le filet (per retem) mais en dessous du filet (sub retem):

Intra retem aues sunt omnigenus, maxime cantrices, ut lusciniolae ac merulae, quibus aqua ministratur per canaliculum, cibus obicitur sub retem $(3,5,14)$.

À l'intérieur du filet, il y a des oiseaux de toutes sortes, surtout des chanteurs, tels que rossignols et merles; on leur fournit de l'eau par un petit canal et la nourriture leur est passée sous le filet.

Pourquoi cette différence? Certains éditeurs, comme Antonio Traglia, ont contourné la difficulté en traduisant sub retem comme s'il s'agissait de per retem, à savoir «attraverso la rete» 3 ; d'autres auteurs ont imaginé que c'était les visiteurs, de Varron, installés pour dîner sur la plateforme (falere) placée au sein de la rotonde qui nourrissaient

427-448; Flach 2004, 137-168; Rodgers 2012, 479-484; Bodel 2012, 45-60, 2012; Jones 2014, 81-812.

3 Traglia 1979, 821. 
les oiseaux par dessous le filet qui entourait la colonnade intérieure de la tholos: c'est l'explication avancée en premier lieu par Georges Seure $^{4}$ et que reprendront tout à tour Lucienne Deschamps ${ }^{5}$ et Robert Etienne6; Seure précise en outre que la qualité du filet intérieur, décrit comme un filet d'oiseleur (rete aviarium), se prêtait bien à ce scénario $^{7}$. Si l'on admet, avec ces auteurs, que l'administration de la pâture dans la rotonde était confiée aux invités de Varron, il faut en déduire que les oiseaux y étaient nourris de façon sporadique; c'est du reste ce que dit indirectement Seure lorsqu'il déclare que:

«L'eau, dans la tholos, et la pâture, sous les portiques, sont l'objet d'un service régulier, fait peut-être par des esclaves (le mot ministratur est employé dans les deux cas)» ${ }^{8}$.

Cet auteur suppose donc que l'eau dans les portiques et la pâture dans la tholos, ne sont pas l'objet d'un service régulier'; on a vu, en effet, que pour l'eau dans les portiques, il n'était pas besoin d'un service extérieur puisque l'eau affluait dans la petite rigole à partir du fleuve proche; quant à la nourriture, l'expression cibus objici-

${ }^{4}$ Des Anges - Seure 1932, 67: «Dans la tholos, la circulation (pedibus circumire) en avant des cages se fait sur une plateforme (falere) qui est elle-même séparée du stylobate par une assise (lapis), si bien que le rebord du stylobate, ou si l'on préfère, le bas du filet, se trouve à environ 3 pieds soit m. 90, au-dessus du niveau de la plateforme sur laquelle se trouvent les visiteurs. Ceux-ci ont donc le bord inférieur du filet au niveau de leurs mains baissées, et il est indiqué que ce soit par là qu'on jette la nourriture aux oiseaux: le geste est ainsi plus naturel et plus commode».

5 Deschamps 1987, 87: «Les personnes se trouvant sur la banquette, à un niveau inférieur par rapport au sol de la volière, font passer la nourriture sous le filet et non à travers le filet»

${ }^{6}$ Etienne 2006, 307: «La pâture ici se donne par en dessous, sub retem, en passant par en dessous de la cordelette qui tend horizontalement les mailles du filet: le bas du filet se trouve environ à un pied plus haut, audessus de la plateforme sur laquelle évoluent les visiteurs; ceux-ci n'ont qu'à jeter naturellement la nourriture au bas du filet qui est au niveau de leurs mains baissées».

${ }^{7}$ Des Anges - Seure 1932, 64: «Le réseau large et fin permettra aux invités l'illusion de croire les oiseaux de la tholos en liberté, et de goûter au centre d'une cage: c'est l'impression recherchée par Varron».

${ }^{8}$ Des Anges - Seure 1932, 66.

${ }^{9}$ Sans doute suppose-t-il que la rigole dans les portiques est alimentée par le fleuve et n'exige donc aucun service. 
tur sub retem doit être interprétée comme l'expression similaire citée plus haut cibus ministratur inter retem, c'est-à-dire qu'elle désigne, dans ce cas également, un agent indéfini qui nourrit régulièrement les oiseaux de la tholos et qui ne semble pas pouvoir être identifié avec d'illustres invités de Varron; il s'agit donc, d'un esclave préposé à la volière, peut-être le puer chargé de faire tourner la table placée au centre de la rotonde (haec ab uno puero qui ministrat, $\underline{3,5,15 \text { ). }}$

Comment le jeune esclave s'y prenait-il pour nourrir les oiseaux de la rotonde? Varron nous dit que l'on pouvait y accéder par un petit sentier qui passait entre les deux bassins (inter eas piscinas tantunmodo accessus semita in tholum, 3, 5, 12); Seure ${ }^{10}$ et Etienne ${ }^{11}$ supposent, à juste titre selon moi, que ce sentier conduisait directement au centre de la tholos, ce qui permettait aux nombreux amis de Varron venus visiter sa volière d'admirer la coupole de la rotonde ornée de Lucifer et d'Hespérus et d'une rose des huit vents semblable à celle de l'artiste Cyrrhus à Athènes (3, 5, §**). Il est difficile, en revanche, d'imaginer que le chemin conduisait dans la cage des oiseaux de la rotonde puisque Varron précise que tout l'espace intérieur était garni de perchoirs formés par des bâtons enfoncés dans chaque colonne et agencés comme les gradins d'un petit théâtre (theatridion avium) ${ }^{12}$; par conséquent, l'esclave n'aurait pu s'y mouvoir et le seul moyen - et le plus simple - de nourrir les oiseaux de cette cage était de le faire par l'extérieur, comme pour les cages des portiques.

Cependant, revenant à notre question principale, on se demande pourquoi l'esclave soulève le filet plutôt que de jeter la nourriture à travers ses mailles. C'est que Varron nous a dit aussi que le filet extérieur était fait de boyaux à petites mailles (reticuli e nervis) pour permettre aux oiseaux, juchés sur les sièges (sedilia avium) de leur théâtre, de voir ce qui se passait dans le bosquet sans toutefois pouvoir s'échapper (Inter columnas exteriores pro pariete reticuli e neruis sunt, ut prospici in siluam possit et quae ibi sunt uideri neque auis ea transire, $3,5,13)$. Il semble donc bien que ce soit le maillage très

10 Des Anges - Seure 1932, 58 n. 48: «Deux colonnettes intérieures entre lesquelles il faudra bien passer pour accéder à l'intérieur de la cage, qui est aussi un lieu de repos et une salle à manger».

${ }^{11}$ Etienne 2006, 306: «Pour gagner la tholos (tholum) on dispose d'un sentier (semita) débouchant sur un passage d'entrée entre deux colonnes de la cage des oiseaux, qui est une salle à manger».

12 Inter has et exteriores gradatim substructum ut theatridion auium, mutuli crebri in omnibus columnis impositi, sedilia auium. 
serré qui ait contraint le puer à soulever le filet extérieur de la cage de la rotonde pour nourrir les oiseaux.

Il restera, pour finir, à décrire la manière dont le puer, chargé de nourrir les oiseaux des cages des portiques et de la rotonde, s'acquittait de la même tâche vis-à-vis des canards; je montrerai que, dans ce cas également, Varron décrit d'abord l'architecture du lieu, puis le genre de volatiles et enfin le mode d'alimentation régulière dispensée aux derniers hôtes de sa volière (convivae $3,5,14)^{13}$ : on comprendra alors pourquoi la volière que Varron avait construite pour son agrément (delectationis causa) avait suscité l'admiration d'un grand nombre de ses amis ${ }^{14}$.

Ginette Vagenheim

\section{Bibliographie}

Becker - Terrenato $2012=$ Roman republican villas, architecture, context, and ideology, edd. J. A. Becker - N. Terrenato, Ann Arbor 2012.

Bodel 2012 = J. P. Bodel, Villaculture, in Becker - Terrenato 2012, 45-60.

Bona - Lévy - Magnaldi 2012 = Vestigia notitiai: scritti in memoria di Michelangelo Giusta, a cura di E. Bona - C. Lévy - G. Magnaldi, Alessandria 2012.

Des Anges - Seure $1932=$ Ch. Des Anges - G. Seure, La volière de Varron, Paris 1932.

Deschamps $1987=\mathrm{L}$. Deschamps, La salle à manger de Varron à Casinum ou 'Dis-moi où tu manges, je te dirai qui tu es', «BSTEC», 191192, 1987, 63-93.

Dunbabin $1996=$ K. M. D Dunbabin, Convivial spaces: dining and entertaintment in the Roman Villa, «JRA », 9, 1996, 66-80.

Etienne 2006 = R. Etienne, La volière de Varron à Casinum (Italie), «REA», 108, 2006, 299-312.

Flach 2004 = D. Flach, Varro's Vogelhaus: Wohlleben im Landleben, «Gymnasium», 111, 2004, 137-168.

Fuchs 1962 = G. Fuchs, Varros Vogelhaus bei Casinum, «MDAI(R)», 69, 1962, 69, 96-105.

${ }^{13}$ J'ai présenté cette dernière analyse lors de la séance publique de la Société des Etudes latines à l'université de Paris IV-Sorbonne, sous le titre suivant: «Qui sont les convivae de la volière de Varron? Une vexata quaestio de l'antiquité à nos jours (11 juin 2016)».

${ }^{14}$ Varr. $R$. R. 3, 5, 4: Duo genera sunt, inquit, ornithonis: unum delectationis causa, ut Varro hic fecit noster sub Casino, quod amatores inuenit multos. 
Green 1997 = C. M. C. Green, Free as a bird: Varro de re Rustica III, «AJPh », 118, 3, 1997, 427-448.

Guiraud 1997 = M. T. Varro, Économie rurale, ed. Ch. Guiraud, III, Paris 1997.

Hoope - Ash 1939 = M. T. Varro, On agriculture, ed. W. D. Hoope - H. B. Ash, Harvard 1934

Jones 2014 = F. M. A. Jones, Roman gardens, imagination and cognitive structure, «Mnemosyne», 67, 5, 2014, 81-812.

Keil 1884-1902 = M. Terenti Varronis rerum rusticarum libri tres, ed. H. Keil, Leipzig 1884-1902.

Sauron 1994 = G. Sauron, Quis deum? L'expression plastique des idéologies politiques et religieuses à Rome à la fin de la République et au début du Principat, Rome 1994.

Rodgers $2012=\mathrm{R}$. H. Rodgers, A few thoughts on the text of Varro's «Res rusticae», in

Vestigia notitiai: scritti in memoria di Michelangelo Giusta, a cura di E. Bona - C. Lévy - G. Magnaldi 2012, 479-484.

Traglia 1979 = Opere di Marco Terenzio Varrone, a cura A. Traglia, di Torino 1979.

Van Buren - Kennedy 1919 = A. Van Buren - R. M. Kennedy, Varro's aviary at Casinum, «JRS», 9, 1919, 59-66. 